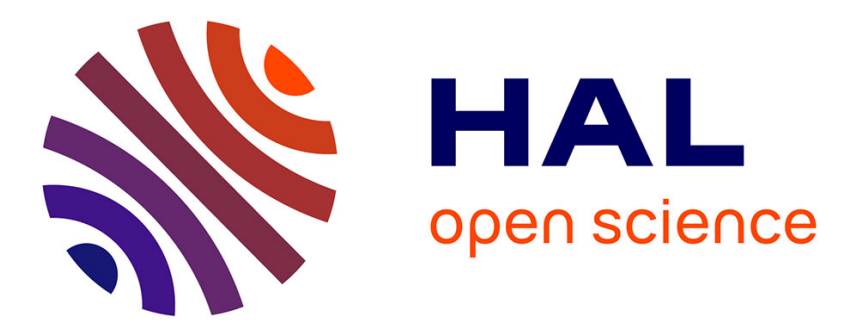

\title{
Magnetization analysis of oriented chains of hexagonal cobalt nanoplates
}

\author{
E. Vargas, J. C. Denardin, R. Lavín, P. Mascaró, Corinne Chanéac, Thibaud
} Coradin

\section{> To cite this version:}

E. Vargas, J. C. Denardin, R. Lavín, P. Mascaró, Corinne Chanéac, et al.. Magnetization analysis of oriented chains of hexagonal cobalt nanoplates. Journal of Applied Physics, 2014, 115 (17), pp.17B521. 10.1063/1.4867345 . hal-01139029

\section{HAL Id: hal-01139029 https://hal.sorbonne-universite.fr/hal-01139029}

Submitted on 3 Apr 2015

HAL is a multi-disciplinary open access archive for the deposit and dissemination of scientific research documents, whether they are published or not. The documents may come from teaching and research institutions in France or abroad, or from public or private research centers.
L'archive ouverte pluridisciplinaire HAL, est destinée au dépôt et à la diffusion de documents scientifiques de niveau recherche, publiés ou non, émanant des établissements d'enseignement et de recherche français ou étrangers, des laboratoires publics ou privés. 


\title{
Magnetization analysis of oriented chains of hexagonal cobalt nanoplates.
}

\author{
E. Vargas ${ }^{1,2,3}$, J. C. Denardin ${ }^{1,2}$, R. Lavín ${ }^{4}$, P. Mascaró ${ }^{1,2}$, C. Chaneac ${ }^{3}$ and T. Coradin ${ }^{3}$ \\ ${ }^{1}$ Departamento de Física, Universidad de Santiago de Chile (USACH), Av. Ecuador 3493, \\ Santiago 9170124, Chile \\ ${ }^{2}$ Centro para el Desarrollo de la Nanociencia y Nanotecnología (CEDENNA), Av. Ecuador \\ 3493, Santiago 9170124, Chile \\ ${ }^{3}$ UMPC Univ Paris 06; CNRS, Chimie de la Matière Condensée de Paris, Collège de \\ France, 75005 Paris, France. \\ ${ }^{4}$ Facultad de Ingeniería, Universidad Diego Portales, Ejército 441, Santiago 8370191, \\ Chile
}

\begin{abstract}
High quality single-crystalline hexagonal cobalt nanoplates have been obtained by a simple chemical reduction method using ultrasonic assistance. Individual nanoplates tend to form agglomerates due to their strong in-plane anisotropy. These particles were incorporated within a polyethylene matrix by a solution blending method and arrays of magnetic chains were generated by solidification of the solution of nanoparticles in the presence of a magnetic field. The easy axis of the Co plates is oriented along the field, in the direction of the chains length. Micromagnetic simulations, magnetic measurements and FORC diagrams are used to clarify the complex magnetic behavior in these systems.
\end{abstract}




\section{INTRODUCTION}

In recent years, controllable synthesis of low-dimensional metallic materials with special geometries has attracted interest due to their diverse potential applications in biomedicine and as novel material for engineering and electronic devices [1]. Nano and submicronparticles with high aspect ratio, particularly of $\mathrm{Fe}$, $\mathrm{Co}$ and $\mathrm{Ni}$ elements, have been studied because controlling the shape and morphology of particles is an effective method for tuning the magnetic properties [2]. In this line, several studies have focused on the simple and large-scale synthesis of regular and periodic magnetic structures [3]. Recently there were various reports in the literature that describe the dispersion of ferromagnetic particles in polymeric matrices by in-situ polymerization, melt compounding and solution blending [46]. This latter method appears to be an attractive route because an external magnetic field provides the possibility of controlling the organization of the particles in the matrix.

In this work, large scale and high quality single-crystalline hexagonal cobalt nanoplates have been obtained by a simple chemical reduction method at room conditions without complex apparatus [7]. These particles were incorporated in a polyethylene matrix by a solution blending method. A complex structure formed by chains of spherical agglomerates of Co platelets forms spontaneously but the direction of these chains can be controlled by solidification of the solution of nanoparticles in the presence of a magnetic field. The easy axis of the Co stacks is oriented along the field, in the direction of the chains length. The collective magnetic properties of this material have been studied by hysteresis loops and using first-order reversal curves (FORC) analysis.

\section{EXPERIMENTAL METHODS}

All reagents were analytical grade and used without further purification. Cobalt nanoplates were synthesized by our previous reported procedure [7]. In brief, $50 \mathrm{uL}$ of cobalt chloride $(0.4 \mathrm{M})$ and $15 \mathrm{~mL}$ of cetyltrimethylammonium bromide $(4 \mathrm{mM})$ were mixed in typical glass vial on ultrasonic bath. Then, $100 \mathrm{uL}$ of hydrazine was dropped in the reaction mixture and the $\mathrm{pH}$ was adjusted to 10 using ammonia solutions. The general redox reaction could be expressed as:

$$
2 \mathrm{Co}^{2+}+\mathrm{N}_{2} \mathrm{H}_{4}+4 \mathrm{OH}^{-} \rightarrow 2 \mathrm{Co}+\mathrm{N}_{2}+4 \mathrm{H}_{2} \mathrm{O}
$$

The final product was purified by magnetic decantation, washed several times and dried. 
The morphological and structural characterization of the samples was carried out by scanning electron microscopy (SEM), in a Zeiss EVO MA10 and transmission electron microscopy (TEM), in a $120 \mathrm{kV}$ Tecnai Spirit G2. The hysteresis loops was measured at room temperature on a vibrating sample magnetometer (VSM). The FORC curves were measured in a homemade Alternating Gradient Force Magnetometer (AGFM).

The nancomposite film was produced by solution blending method. First, the Co nanoplates were dispersed into $1 \mathrm{~mL}$ of toluene. After that, $100 \mathrm{uL}$ of black colloidal system was incorporated into the dilution of polyethylene-toluene $(10 \% \mathrm{wt})$. Finally, the mixture was supported on a square glass and vacuum dried under an external magnetic field of 2500 Oe. Micromagnetic simulations were performed using the 3D OOMMF package [8]. Under this frame, the ferromagnetic system is divided into cubic cells with a uniform magnetization inside each cell. For our simulations we use the typical Cobalt parameters: saturation magnetization $M_{s}=1400 \times 10^{3} \mathrm{~A} / \mathrm{m}$, exchange stiffness constant $A=30 \mathrm{~J} / \mathrm{m}$, and a mesh size of $3 \mathrm{~nm} \times 3 \mathrm{~nm} \times 5 \mathrm{~nm}$, where spins are free to rotate in three dimensions. In all the cases the damping constant is 0.5 .

\section{RESULTS AND DISCUSSION}

Figure 1(a), shows the SEM image and reveals micron-long chains formed by the lineal organization of small particles. The magnified image (inset) shows particles with irregular shape and faceted surfaces, suggesting the existence of agglomerated nanoplates. To visualize details, a dilute colloidal solution was used to prepare TEM samples, as shown in Figure 1(b). This image shows perfect hexagonal particles. The size distribution was determined by processing 387 particles in several micrographs and has been adjusted by Gauss distribution, obtaining an average size about $300 \mathrm{~nm}$. The insert of Figure 1(b) corresponds to the selected area electron diffraction (SAED) and shows the spots of mono crystalline hexagonal particle in $0001 \mathrm{hcp}$ axis zone.

It was observed that the nanoparticles tend to agglomerate and to form long chains (see Figure 1 (a)) due to their strong remanent magnetization and dipolar interactions. In fact, the aspect ratio of the nanoparticles and the strong magnetic anisotropy make them very sensitive to an external magnetic field and it is also possible to artificially generate arrays of magnetic chains by solidification of the solution of nanoparticles in the presence of 
magnetic field in wax or other polymers. Using this approach we generated nanocomposite films of Co nanoplates and polyethylene that were prepared in the presence of external field (oriented chains) and without field (not-oriented chains).

The magnetic properties of the samples were characterized with hysteresis loops as shown in Figure 2 (a) for not-oriented chains and oriented chains with field. The samples that formed micro chains were measured with the field parallel and perpendicular to the chains. From Figure 2 (a) one can see that the disordered Co nanoplates (not-oriented chains) have a coercivity of 280 Oe and a remanent magnetization of 0.2 . Figure 2 (a) shows that there is a clear anisotropy, or easy magnetization axis, in the direction of the chains aligned by the external field. This means that it is easier to reach saturation when the field is applied in the direction of the chains than in the perpendicular direction. The coercivity values of the sample measured parallel and perpendicular to the chains are 150 Oe and 230 Oe respectively.

In order to infer the magnetic behavior of these hexagonal nanoplates and how the arrays of nanoplates would tend to agglomerate we have performed micromagnetic simulations, obtaining the coercivity and remanence of an isolated nanoplate with a diameter of $300 \mathrm{~nm}$, with the magnetic field applied in different angles $\theta$ with the z-axis (the out of plane axis).

Figure 2 (b) shows the coercivity and remanence from simulated curves as a function of angle $\theta$ between the $z$-axis and the external magnetic field. Because the easy axis is in the $x y$ plane of the nanoplate, it is expected that when $\theta$ decreases the coercivity increases, as shown in Figure 2 (b). One can see that the coercivity is strongly dependent on the out-ofplane orientation of the nanoplates with respect to the external magnetic field, varying from 306 to 915 Oe. The in-plane remanence of the nanoplate is more than two times higher than the out-of-plane remanence. This means that the individual nanoplates will tend to form agglomerates due to their strong in-plane remanent field, stacking individual hexagonal nanoplates to generate almost spherical agglomerates, as observed in the SEM image of Figure 1. When these agglomerates of nanoplates are dried in presence of the magnetic field, their easy axis (the $x y$ plane) will be oriented in the direction of the field, and consequently along the length of the chains, and the $z$-axis of the nanoplates can be oriented randomly in any direction perpendicular to the length of the chains. 
In order to understand in more details the mechanism of magnetization reversion on these systems we performed a more detailed magnetic analysis using the technique of FORC diagrams [9-12]. This technique is already being used to study different aspects of magnetic systems [12-14].

Figure 3 shows the FORCs (a to c) and the corresponding diagrams ( $d$ to $f$ ) for the samples with not-oriented chains, and the oriented chains measured parallel and perpendicular to the field. The FORCs resemble the hysteresis curves of Figure 2 with the difference that now the complete internal area of the curves are filled with reversal curves. From Figure 3 it is worth noting that the FORCs for the not-oriented chains and the oriented chains measured in perpendicular direction do not present any visible difference (Figure 3 (a) and (c)). On the other hand, the curve for oriented chains measured parallel is clearly different, being more vertical.

The diagrams of Figure 3 ( $\mathrm{d}$ to $\mathrm{f}$ ) show the distribution of coercivity fields $\left(H_{c}\right)$ and interaction fields $\left(H_{i}\right)[13,15]$, and allow one to perform a more detailed analysis of the magnetization reversal mechanisms that take place in these three configurations of the nanoplate arrays. The diagram for the not-oriented chains (Figure 3 (d)) shows two distinct areas (sometimes called magnetic phases) that are more intense. One of this areas is centered at $H_{c}=0$, that is attributed to the reversible magnetization contribution. This contribution is usually associated to the presence of a low coercivity reversible magnetic phase, and in this case could represent the contribution of the Co nanoplates that are in chains perpendicular to the field, or that have their hard axis (z plane) in the field direction. This reversible magnetization component can be observed in the FORC curves of figures 3 (a) as the positive slope at the beginning of each FORC curve.

The other region of the diagram is a small spot centered in $H_{c}=0.28 \mathrm{kOe}$, which represents the irreversible magnetic phase due to the nanoplates or arrays of nanoplates that reverse completely their magnetization with the external field, reaching a new equilibrium magnetization. One can also observe that this region presents a broad distribution of $H_{c}$, as a consequence of a distribution of nanoparticle size and/or a distribution of orientation of the nanoparticles with respect to the external field. The effect of interaction among the nanoparticles can be appreciated by the width of the distributions in the vertical axis, the $H_{i}$ distribution. 
The diagram obtained for the oriented chains measured parallel to the field (see Figure 3(e)) shows only one region of irreversible magnetization centered at $H_{C}=0.25 \mathrm{kOe}$. The size of this magnetic phase is smaller than in the disordered sample (Figure $3(\mathrm{~d})$ ), both in $H_{c}$ and in $H_{i}$. As the physical size distribution of the nanoparticles is the same in both samples, this difference in the value of $H_{c}$ distribution could be attributed to the smaller distribution of easy axis ( $x y$ planes) in the direction of the chains, as compared to the not-oriented sample. The fact that the reversible magnetic phase is not present is another indication of the bistability of the oriented chains generated by the external field in the sample preparation process.

When the oriented arrays of nanoparticles are measured in the perpendicular direction the FORCs are very similar to the ones obtained for the disordered sample (see Figure 3 (a) and (c)). However, the diagrams show distinct features, as shown in Figure 3 (d) and (f). The main difference is the presence of two small spots superimposed in the main irreversible magnetic phase. The first spot is in centered at the same $H_{c}$ value of Figure 3 (e), and the second spot spreads over a high field region, giving in average the same $H_{c}$ distribution than in Figure 3 (d). The $H_{i}$ distribution is smaller than in Figure 3 (d) and the reversible component appears again in this diagram, as a consequence of the fact that the $z$-axis of the nanoplates are oriented randomly in any direction perpendicular to the length of the chains. From the previous results one can conclude that when the oriented chains are formed, very few nanoplates are oriented with their hard axis (z-axis) in the direction of the chains, resulting in a more vertical magnetization curve, with a small coercive field. When the magnetization curve is measured perpendicular to the chains, the magnetization reversal occurs through nanoplates that are oriented at different angles $\theta$, increasing the coercivity of the system.

\section{CONCLUSION}

Based on the analysis of the different magnetic cooperative mechanisms, one can identify different magnetic entities that are present in the here-described Cobalt nanoplates systems. The simplest one is composed by the individual magnetic hexagonal Co platelets. The magnetic behavior of these individual hexagons cannot be observed by macroscopic measuring techniques as their strong magnetic anisotropy and aspect ratio induce their 
agglomeration in almost spherical particles. The more complex structure is the chain of spherical agglomerates, that also forms spontaneously but whose direction can be controlled by solidification of the solution of nanoparticles in the presence of magnetic field in a polymer. The easy axis of the Co stacks are oriented along the field, in the direction of the chains length. Micromagnetic simulations and the careful analysis of the magnetization curves and the FORC diagrams are particularly useful to investigate the collective magnetic properties of these systems.

\section{ACKNOWLEDGMENTS}

The support from Fondecyt grants 1110252 and 11110130; Millennium Science Nucleus, Basic and Applied Magnetism Grant NP10-061-F, AFOSR FA9550-11-1-0347 and CONICYT BASAL CEDENNA FB0807, is gratefully acknowledged.

\section{REFERENCES}

[1] D.V. Talapin, J.S. Lee, M.V. Kovalenko and E.V. Shevchenko. Chemical Reviews 110, 389-458 (2010).

[2] R. Xu, T. Xie, Y. G. Zhao and Y. D. Li. Crystal Growth \& Design 7, 1904-1911 (2007).

[3] S. Behrens. Nanoscale 3, 877-892 (2011).

[4] Q. He, T. Yuan, J. Zhu, Z. Luo, N. Haldolaarachchige, L. Sun, A. Khasanov, Y. Li, D. P. Young, S. Wei and Z. Guo. Polymer 53, 3642-3652 (2012).

[5] T. Wen and K. M. Krishnan. J. Phys. D: Appl. Phys. 44, 393001 (2011).

[6] D. Younesi, R. Mehravaran, S. Akbarian and M. Younesi. Materials and Design 43, 549-559 (2013).

[7] E. Vargas, P. Toro, J.L. Palma, J. Escrig, C. Chaneac, T. Coradin, J.C. Denardin, Materials Letters 94, 121-123 (2013).

[8] M. J. Donahue, D. G. Porter, OOMMF User's Guide, Version 1.2a3 http://math.nist.gov/oommf (2002).

[9] C. R. Pike, A. P. Roberts, K. L. Verosub. Journal of Applied Physics 85, 6660-6667 (1999). 
[10] C. R. Pike. Physical Review B 68, 104424 (2003).

[11] R. Lavín, C. Farías, J. C. Denardin. Journal of Magnetism and Magnetic Materials 324, 1800-1803 (2012).

[12] R. K. Dumas, C. P. Li, I. V. Roshchin, I. K. Schuller, K. Liu. Physical Review B 75, 134405 (2007).

[13] R. Lavín, J. C. Denardin, J. Escrig, D. Altbir, A. Cortés, H. Gómez. IEEE Transactions on Magnetics 44, 2808-2811 (2008).

[14] H. Chiriac, N. Lupu, L. Stoleriu, P. Postolache, A. Stancu. Journal of Magnetism and Magnetic Materials 316, 177-180 (2007).

[15] G. Bertotti, Hysteresis in Magnetism, Academic Press, NY, USA, 1998.

\section{FIGURE CAPTIONS:}

Figure 1: (a) Panoramic and magnified SEM images of chains of agglomerated nanoplates.

(b) TEM image and SAED pattern of the hexagonal nanoplates in hcp crystalline phase.

Figure 2: (a) Magnetization curves normalized by $\mathrm{M}_{\mathrm{s}}$ of Co chains in polymer, and measured as shown in the inset draws. (b) Coecivity and reduced remanence obtained from micromagnetic simulation of one hexagonal Co particle, as function of the angle $\theta$ between the external field and the $\mathrm{z}$-axis (see the inset).

Figure 3: FORCs measured for the three configurations: (a) not-oriented chains, (b) oriented chains with the external field parallel and (c) perpendicular to the chains. The corresponding FORC diagrams for the three configurations are shown in ( $d$ to $f$ ). 

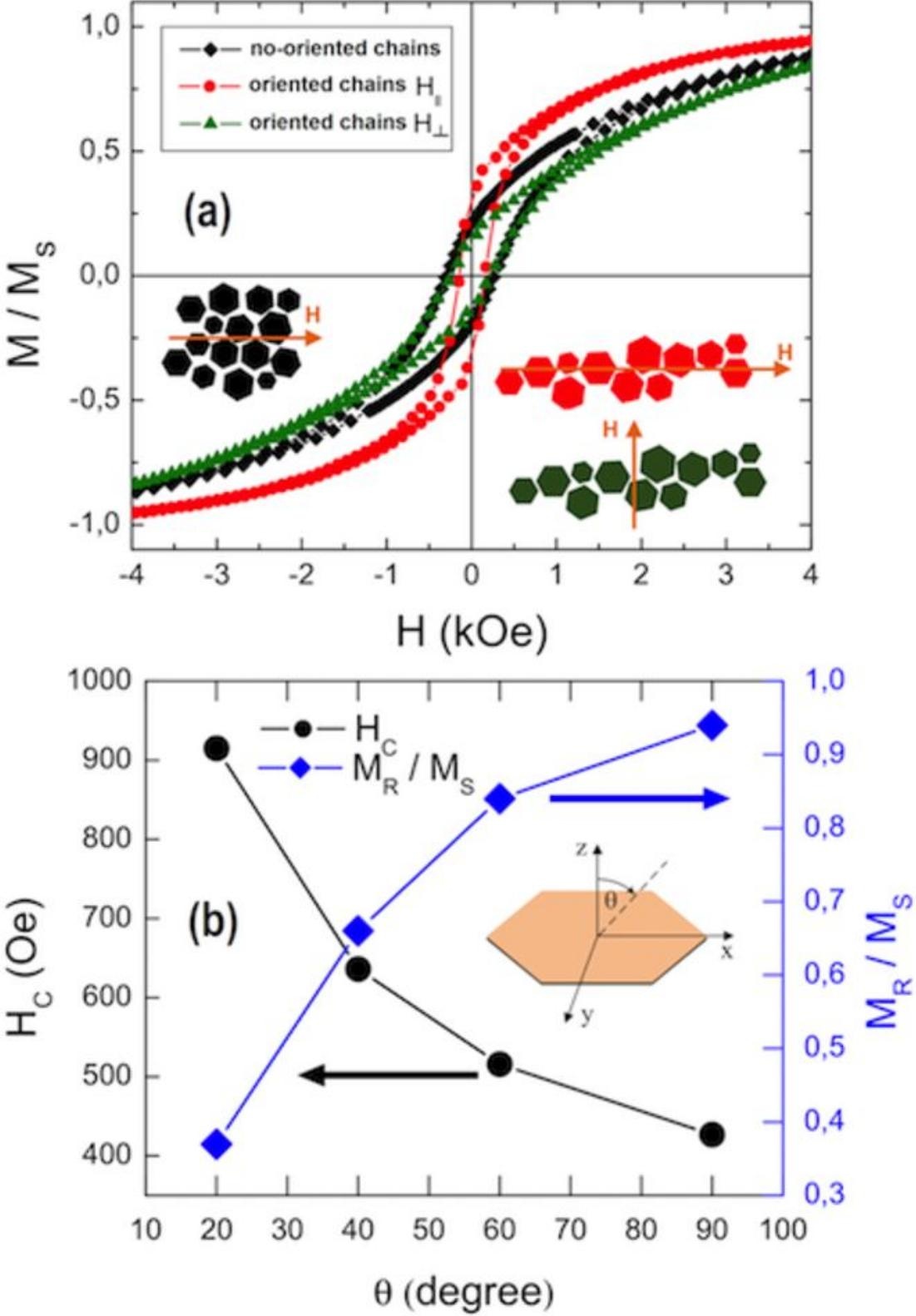

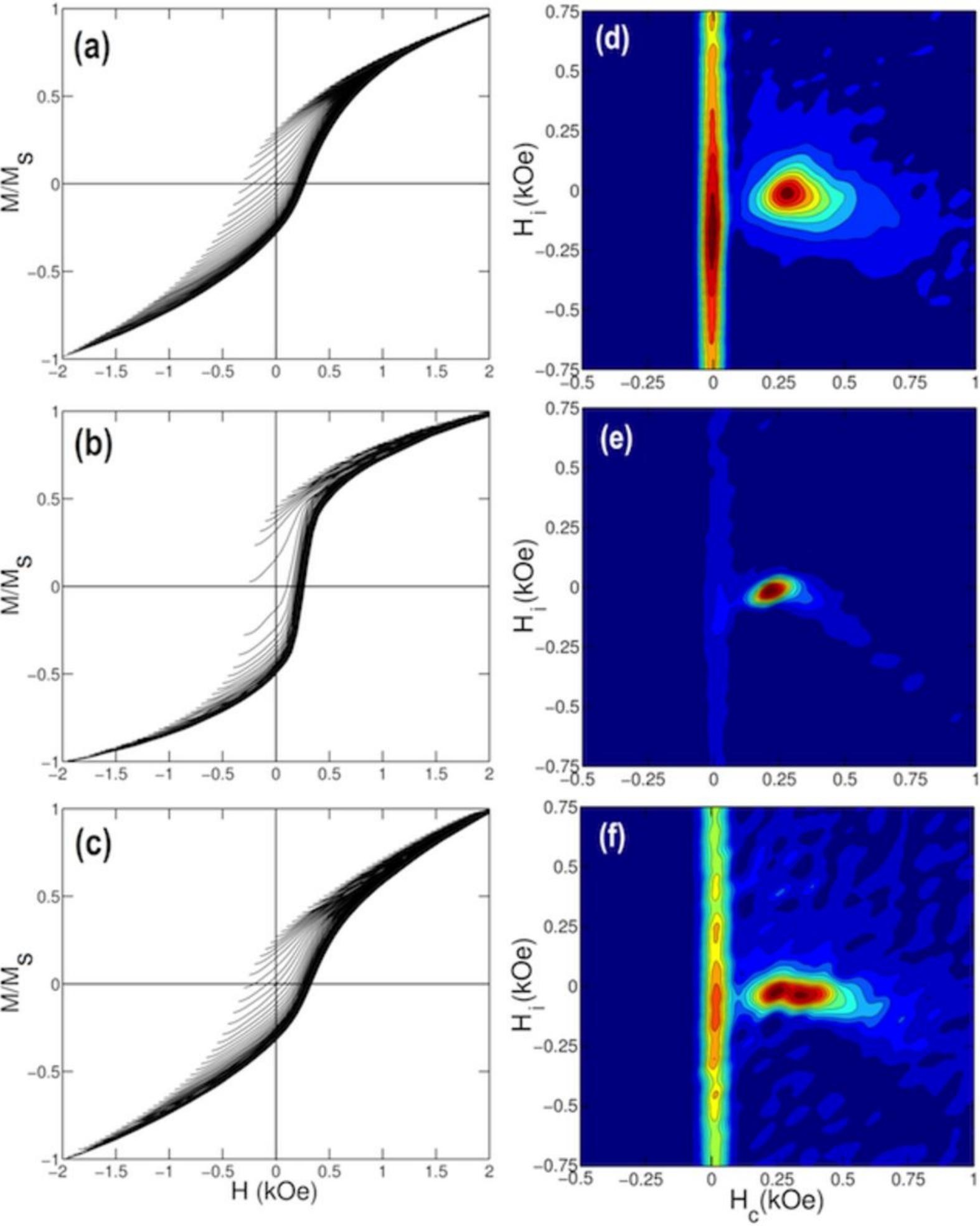

0.75

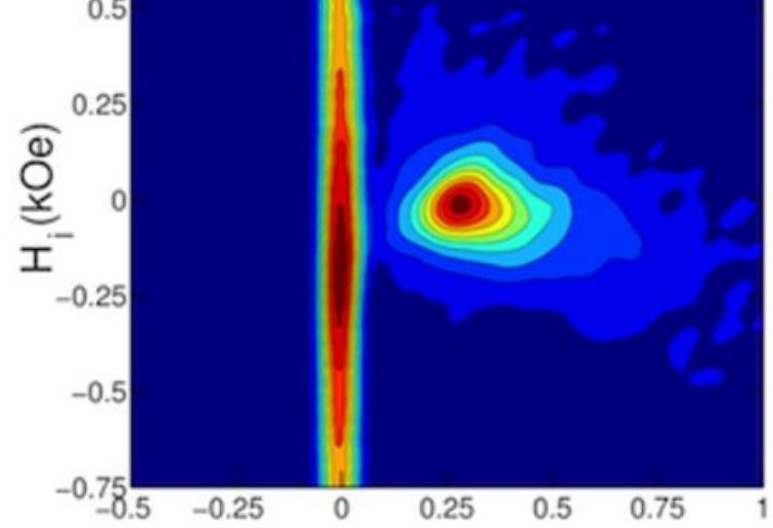

(d) 